Gastritol ${ }^{\circledR}$ Liquid: Leichte Verdauungsbeschwerden natürlich behandeln

\section{Aktuelle Studie bestätigt Wirksamkeit und Verträglichkeit}

Das pflanzliche Arzneimittel Gastritol ${ }^{\circledR}$ Liquid hat in einer nichtinterventionellen Studie [1] mit 149 Patienten seine Wirksamkeit und Verträglichkeit eindrucksvoll unter Beweis gestellt. Die aktuell in der Fachzeitschrift «Phytotherapy Research» veröffentlichte Arbeit schloss Patienten mit leichten Verdauungsbeschwerden oder leichten krampfartigen Beschwerden im MagenDarm-Trakt ein und wies eine sehr gute Ansprechrate von $90 \%$ auf. Dabei verbesserten sich die am stärksten belastenden Symptome um bis zu 67\% - ähnlich stark wie bei synthetischen Präparaten. Die Daten der Untersuchung belegen zudem eine ausgezeichnete Verträglichkeit für Gastritol Liquid: Bei mehr als 2000 definierten Tagesdosen zeigten lediglich 7 Patienten geringfügige Nebenwirkungen. Auch bei den 59 an der Studie beteiligten Diabetikern erwies sich das pflanzliche Präparat bei der Behandlung von Verdauungsstörungen als wirksam. Die erfassten Symptome, unter anderem anhaltendes Völlegefühl, Übelkeit, Würgereiz und Blähungen, verbesserten sich in beiden Studiengruppen im Arzturteil um 30-40\% und um 30-67\% in der Patientenbewertung. Darüber hinaus steigerte sich auch die durch die Symptome beeinträchtigte Lebensqualität, insbesondere in den Bereichen (Arbeits-)Leistung und Schlaf. Gastritol Liquid vereint die Wirkspektren von 6 gastral wirksamen Pflanzenextrakten aus Kamillenblüten, Gänsefingerkraut, Süßholzwurzel, Angelikawurzel, Benediktenkraut und Wermutkraut. Diese haben eine adstringierende, antiphlogistische und krampflindernde Wirkung.
Zudem wird die Verdauung angeregt und die Durchblutung der Magenschleimhaut verbessert. Die Kombination pflanzlicher Wirkstoffe führt zu einem Synergieeffekt: So entfalten Kombinationspräparate eine deutlich stärkere Wirkung als Präparate mit lediglich einem Pflanzenwirkstoff [2]. Gastritol Liquid ist somit eine wirksame und sichere Behandlungsoption bei dyspeptischen Beschwerden, die auch ergänzend zu eventuell notwendigen Veränderungen der Ernährungsgewohnheiten empfohlen werden kann.

\section{Literatur \\ 1 Wegener T et al.: Phytother Res 2015 (Epub ahead of print) 2 Wagner $\mathrm{H}$ et al.: Phytomedicine 2009;16:97-110.}

Weitere Informationen bei

Dr. Gustav Klein GmbH \& Co. KG

www.klein-naturarznei.de

\section{Bauchfett als Drüse}

\section{Silent Inflammation}

Bei den überzähligen Fettzellen handelt es sich um hormonaktives Drüsengewebe, das unseren Gesundheitszustand massiv negativ beeinflusst. Leber, Bauchspeicheldrüse, Schilddrüse und Hypophyse sind als hormonbildende Organe bekannt. Dass das reine Bauchfett auch Drüsenfunktion hat, ist vielen Patienten nicht bewusst.

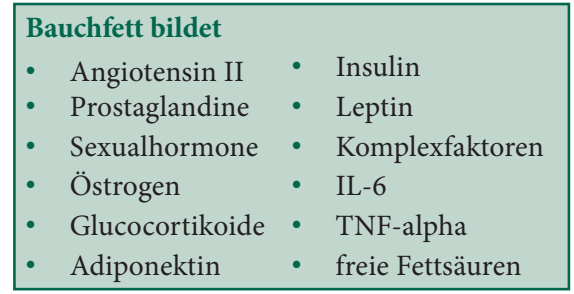

Durch die proinflammatorischen Zytokine und Hormone können unterschiedliche, teilweise diffuse und nicht organbezogene Beschwerden ausgelöst werden.

Silent Inflammation bezeichnet die vermehrte Ausschüttung von proinflammatorischen Zytokinen und, damit verbunden, das Auftreten von chronischen Erkrankungen. Dieser Zustand der leichten systemischen und chronischen (subklinischen) Entzündung unterscheidet sich erheblich von dem einer akuten Entzündung.

Subklinische chronische Entzündungen stehen in engem Zusammenhang oder interagieren mit chronischem Stress, Adipositas, Parodontitis/apikaler Parodontitis, chronischen Virusinfekten, Autoimmunerkrankungen, Nahrungsmittelunverträglichkeiten, Darmschleimhautentzündungen, generalisierter Arteriosklerose, Schlafapnoe-Syndromen, De- menz, Nikotinbusus und Bluthochdruck. Oft ist hier unklar, was Ursache und was Wirkung ist. Werden die oben genannten Diagnosen gestellt, liegt immer eine subklinische Entzündung vor.

Ziel der therapeutischen Arbeit sollte sein, alle Erkrankungen zu erkennen, bei denen proinflammatorische Zytokine gebildet werden, um mögliche additive Effekte zu unterbrechen.

Mit freundlicher Unterstützung von Dr. med. Carsten Nolte, Kardiologe

Weitere Informationen bei VITATEC Medizintechnik GmbH deutschland@vitatec.com www.vitatec.com

\title{
Ticker+++ Ticker+++ Ticker+++ Ticker+++ Ticker+++ Ticker+++ Ticker+++
}

Dr. Loges. Die für körperliche und geistige Leistungsfähigkeit wichtigen B-Vitamine werden in vitamin $B$-loges ${ }^{\circledR}$ komplett mit Pangamsäure (Vitamin B15) ergänzt. Durch diese Spezialkombination werden B-Vitamine in ihrer Wirkung optimiert und der Organismus besser mit Sauerstoff versorgt. Informative Patienten-Broschüren, eine Präparatekarte und ein Sonderdruck zum Supervitalstoff Pangamsäure sind kostenfrei bei Dr. Loges abrufbar.

Dr. Loges GmbH + Co. GmbH

Heidi Franke

franke@loges.de
hypo-A. Aus Kaltwasserfischen gewonnene Öle sind ein wahrer Fundus an essenziellen Omega-3-Fettsäuren, die der menschliche Körper nur sehr begrenzt herstellen kann. Empfohlen werden daher im Allgemeinen 2 bis $3 \mathrm{Fi}-$ schmahlzeiten pro Woche. Der Bedarf an Omega-3-Fettsäuren kann zudem über Fischölkapseln gedeckt werden. Lachsölkapseln von hypo-A enthalten Öl von Kaltwasserfischen aus dem Atlantik und Pazifik, das besonders rein und reich an Omega-3-Fettsäuren ist.

$$
\begin{aligned}
& \text { hypo-A GmbH } \\
& \text { info@ hypo-a.de } \\
& \text { www.hypo-a.de }
\end{aligned}
$$

WÖRWAG. Bis zu $70 \%$ der Patienten mit chronischem Nierenversagen leiden an einer Nervenschädigung. Bei diesen Patienten könnte eine ergänzende Behandlung mit dem vitaminähnlichen, antineuropathischen Wirkstoff Benfotiamin von Nutzen sein. Darauf weist ein internationales Wissenschaftlerteam um Professor István Wittmann, Universität Pécs, Ungarn, in Ausgabe 4/2015 der Fachzeitschrift «Diabetes, Stoffwechsel und Herz» hin.

Wörwag Pharma GmbH \& Co.KG

info@woerwagpharma.com www.woerwagpharma.de 


\section{News}

Forsch Komplementmed 23 | 1 | 16

Wenn sich das Immunsystem gegen Umweltstoffe wehrt

\section{Allergische Rhinokonjunktivitis mit hoher Prävalenz in Deutschland}

Jeder vierte bis fünfte Deutsche ist von einer allergischen Erkrankung betroffen [1]. Dabei dominiert das Thema vor allem die Frühlingsmonate, die von einem intensiven Pollenflug geprägt sind. So machen Menschen mit allergischer Rhinokonjunktivitis (Heuschnupfen) mit rund 86\% den größten Anteil der AllergiePatienten aus [1]. Sie leiden unter Symptomen wie Niesen, Sekretion, juckenden und tränenden Augen - aber auch Halsbeschwerden können sich einstellen. Gegen trockene und gereizte Schleimhäute im Mund- und Rachenraum können die isla ${ }^{\circledR}$ Halspastillen helfen. Sie werden in der Offizin auch häufig begleitend zur Einnahme von Antihistaminika, deren Einsatz zu Mundtrockenheit führen kann, empfohlen.
Bei Allergien handelt es sich um entzündliche Prozesse, die vor allem die Haut, den Darm und die einer Abwehrreaktion des Immunsystems gegen eigentlich harmlose Allergene (Umweltstoffe) wie Pollen, Hausstaubmilben, Tierhaare und Nahrungsmittel. Aufgrund ihrer hohen Prävalenz von 20-30\% haben sich Allergien in Deutschland zu einer Volkskrankheit entwickelt - die Tendenz der Neuerkrankungen ist dabei steigend. Eine allergische Rhinokonjunktivitis gilt neben der Neurodermitis als die häufigste Form der allergischen Erkrankung: So zeigen Zahlen der Deutschen Gesellschaft für Allergologie und Klinische Immunologie (DGAKI), dass in Deutschland rund $86 \%$ der Allergie-Patienten unter einer Pollenallergie leiden [1]. Atemwege betreffen. Dazu kommt es aufgrund

\section{Auch auf Kreuzallergien achten}

Das hohe Vorkommen allergischer Erkrankungen muss oftmals auch bei Therapieempfehlungen in anderen Indikationen berücksichtigt werden. Denn meist ist es nicht nur ein Allergen, das die Symptomatik auslöst: Eine gewisse Ähnlichkeit in der Oberflächenstruktur der Proteine lässt das Immunsystem auch bei anderen Allergenen überreagieren und sorgt für Kreuzallergien. Das macht ein gezieltes Nachfragen bei den Patienten sowie eine sorgfältige Präparate-Empfehlung notwendig.

In Bezug auf die Pollenallergie geht man davon aus, dass die Hälfte der Patienten zusätzlich auf bestimmte Lebensmittel oder Inhaltsstoffe in Arzneimitteln reagiert. Ein Beispiel dafür ist Thymian, der etwa im Rahmen von Atemwegsinfekten zum Einsatz kommt: Thymianhaltige Hustenmittel bergen für Birkenpollenallergiker das Risiko einer Kreuzallergie und sind bei Vorliegen

einer allergischen Grunderkrankung entsprechend kontraindiziert [2].
Für Allergiepatienten, die zusätzlich unter Beschwerden wie akuten oder
chronisch-entzündlichen Atemwegsinfekten mit Husten leiden, hat sich der
sehr gut verträgliche Efeu-Spezial-Extrakt EA $575^{\circledR}\left(\right.$ Prospan $\left.{ }^{\circledR}\right)$ als Husten-
mittel der Wahl bewährt. Für das rationale Phytopharmakon sind bisher
weder Kreuzallergien noch Wechselwirkungen bekannt. Mit der 4-fach-Kraft
des Efeus lindert Prospan effektiv verschiedene respiratorische Beschwerden:
Es löst den festsitzenden Schleim, fördert das Durchatmen und lindert in
Folge dessen den Hustenreiz. Für einzelne Bestandteile des Efeu-Spezial-Ex-
trakts EA 575 sind anti-entzündliche Effekte bekannt [3].

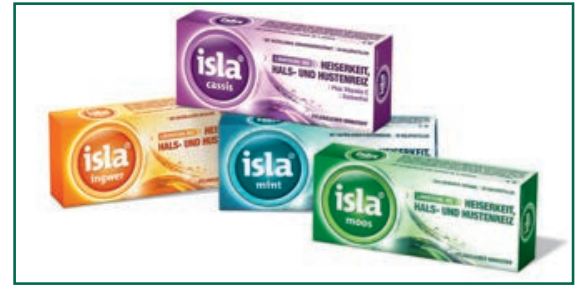

Das Beschwerdebild einer allergischen Rhinokonjunktivitis ist vielfältig: Die Schleimhäute in Mund- und Nasenbereich sind geschwollen und trocken und reagieren mit Schnupfen und Juckreiz, der oftmals auch die Augen betrifft und zu deren Rötung sowie Tränen führen kann. Folge der trockenen Schleimhäute in Mund- und Rachenraum können ein Kratzen im Hals sowie Räusperzwang sein. Entsprechend sollte deren intensive Befeuchtung sichergestellt werden. Die isla Halspastillen mit dem Spezialextrakt aus Isländisch Moos unterstützen hier zweifach: Mit seinem hohen Schleimstoffgehalt befeuchtet das pflanzliche Isländisch Moos die Schleimhäute und überzieht diese zugleich mit einem Schutzfilm, der sie vor weiteren äußeren Reizen bewahrt. Das sorgt für eine sanfte Linderung der Beschwerden. isla hat sich zudem begleitend zur Einnahme von Antihistaminika, Standardtherapeutika vieler Pollenallergiker, bewährt: Denn ihr Einsatz kann u.a. zu Xerostomie (Mundtrockenheit) führen - ein Beschwerdebild, für das isla ein sinnvolles, gut verträgliches Mittel darstellt.

\section{Literatur}

1 Renz H et al.: Allergieforschung in Deutschland. Ein Atlas mit Bestandsaufnahme, Defizit- und Bedarfsanalyse. Marburg, Deutsche Gesellschaft für Allergologie und Klinische Immunologie (DGAKI), 2008.

2 www.fachinfo.de/suche/thymian

3 Lang C et al.: Planta Med 2015;81:968-974.

Weitere Informationen bei

Engelhard Arzneimittel GmbH \& Co. KG www.engelhard.de

\section{Neuer ISCADOR-Vertrieb}

Die Iscador AG hat am 1. November 2015 den Vertrieb von ISCADOR in Deutschland von der Weleda AG übernommen. Alle medizinischen und wissenschaftlichen Fragen werden seit diesem Zeitpunkt von der Iscador AG beantwortet. In der Schweiz ist dieser Schritt bereits zum 1. Februar 2015 vollzogen worden. Das ISCADOR-Sortiment ist unverändert geblieben und auch an den Bezugsquellen sowie den Pharmazentralnummern hat sich nichts geändert.

Die Iscador AG ist eine Aktiengesellschaft mit Sitz in Arlesheim in der Schweiz, welche sich im Besitz des gemeinnützigen Verein für Krebsforschung, Arlesheim, und der gemeinnützigen Gesellschaft für klinische Forschung e.V., Berlin, befindet. Es existiert eine Niederlassung gleichen Namens in Deutschland. Kernaufgaben der Iscador AG sind die Produktion und der Vertrieb des seit Jahrzenten vom Verein für
Krebsforschung hergestellten Mistelpräparates ISCADOR sowie die Forschung und Entwicklung im Bereich der Integrativen Onkologie. Die kontinuierliche Versorgung der Patienten mit ISCADOR ist selbstverständlich weiterhin gewährleistet. Alle Therapieanfragen zu ISCADOR werden seit dem 1. November 2015 von der Iscador AG bearbeitet.

Infoline für Fachkreise: E-Mail: infoline@ iscador.de Tel: 08007067000 (kostenlos) Mo-Fr, 10:00-13:00 Uhr

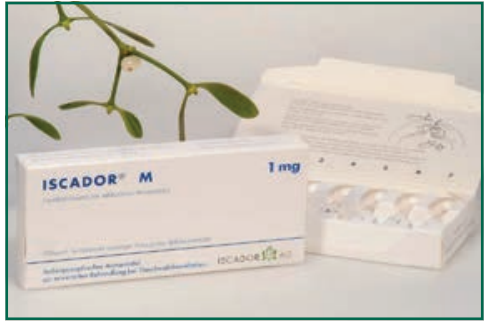


Integrative Onkologie

\section{Nach dem 6. Mistelsymposium ...}

... ist vor dem 7. Mistelsymposium. Unmittelbar nach dem Erfolg des 6. Mistelsymposiums, das unter der Leitung des Autors dieses Artikels vom 12.-14. November 2015 mit mehr als 100 in- und ausländischen Wissenschaftlern und Ärzten verschiedener Fach- und Therapierichtungen stattfand (siehe Nachbericht in Forsch Komplementmed 6/2015), wurde bereits der Termin für das 7. Mistelsymposium festgelegt: 7.-9. November 2019 in der Europäischen Akademie Otzenhausen, Nonnweiler/Saarland. Details zu Ankündigung, Programm, Abstracts und Bücher der vergangenen Mistelsymposien sind unter www.mistelsymposium.de zu finden.

Die Mistelsymposien finden alle 4 Jahre statt. Daher wird nach einem Symposium nicht nur das Buch mit den Langfassungen aller Beiträge zusammengestellt (und im KVC-Verlag, Essen, voraussichtlich Ende dieses Jahres als Printund als Online-Version erscheinen), sondern auch der Termin der Folgeveranstaltung festgelegt.

Die Veranstalter wollen die Mistelsymposien als ein integratives Element in der Onkologie verstanden wissen. Denn mehr als die Hälfte aller Tumorpatienten im deutschsprachigen Raum verwenden Mistelpräparate im Rahmen ihrer Therapie auf Basis ärztlicher Verschreibung. Deshalb ist es wichtig, die therapeutischen Möglichkeiten dieser Arzneipflanze und ihre Inhaltsstoffe noch besser kennenzulernen und damit für die Patienten und deren Tumortherapie nutzbar zu machen. Es sollen Brücken geschlagen werden zwischen den Therapierichtungen, zwischen Pharmazie und Medizin, zwischen Grundlagenforschung und
Anwendung und besonders zwischen konventioneller und komplementärer Onkologie. Daher werden die Mistelsymposien von zahlreichen medizinischen und pharmazeutischen Fachgesellschaften zusammen mit der Karl und Veronica Carstens-Stiftung veranstaltet. Darüber hinaus wird angestrebt, dass sich im Rahmen der Mistelsymposien Fachgesellschaften und Vertreter sowohl der komplementären als auch der konventionellen Onkologie wissenschaftlich mit Fragen und Studien zur sicheren und verlässlichen Anwendung der Mistel in der Tumortherapie beschäftigen.

Dr. Rainer Scheer

Weitere Informationen

Dr. Rainer Scheer

Carl Gustav Carus-Institut

Tel. 0723368418

rainer.scheer@carus-institut.de

\section{Zink und basische Bicarbonate helfen, den Körper zu entlasten}

\section{Fastenkuren können zu Übersäuerung führen}

Nicht nur religiöse Menschen entscheiden sich für eine Fastenkur. Meistens sind es Frauen, die 1- bis 2-mal im Jahr für 1 Woche auf jegliche feste Nahrung verzichten. Wer fastet, führt dem Körper lediglich Wasser, Tee, Fruchtsaft oder Gemüsebrühe zu. Bei Fastenden kann der Nahrungsentzug zu einem Gefühl von tiefer Zufriedenheit und zu neuer Energie führen. In der Erfahrungsmedizin werden Fastenkuren zur inneren Reinigung und Entschlackung bis hin zum Heilfasten als Therapie gegen Zivilisationskrankheiten wie Allergien oder Bluthochdruck eingesetzt. Doch Achtung: Im Hungerzustand entstehen im Körper vermehrt Ketosäuren. Ähnlich wie Straßen mit hohem Verkehrsaufkommen «verstopft» auch das Gewebe des Körpers, wenn zu viele Säureteilchen anfal- len. Bei einem Ausscheidungsstau verbleiben somit die Säuren im Bindegewebe und belasten die «Puffersysteme» des Körpers. Um die «Pufferkapazität» des Blutes zu erhalten, springen Knochen und Muskeln als Säurepuffer ein. Das bleibt nicht ohne Folgen: Muskeln büßen Substanz ein, Knochen verlieren Mineralien.

Mithilfe der 21 Teststreifen, die jeder Packung BASENTABS pH-balance PASCOE ${ }^{\circledR}$ beiliegen, kann der Fastende bequem feststellen, wie sich die Fastenkur auf den pH-Wert des Körpers auswirkt: Einen der Teststreifen in den Urin halten und die entstandene Farbe mit der Farbskala vergleichen. Mehrmals am Tag gemessen, ergibt sich im Idealfall eine Kurve, die wie ein großes «M» aussieht. Liegen nahezu alle im Tagesverlauf gemessenen $\mathrm{pH}$-Werte im sauren Bereich, ist der Körper vermutlich übersäuert. Während einer Fastenkur kann es somit hilfreich sein, den Körper mit einem mineralischen Nahrungsergänzungsmittel zu entlasten. Präparate mit Zink sind dabei besonders wertvoll für einen ausgeglichenen Säure-Basen-Haushalt. BASENTABS pH-balance PASCOE enthalten neben Zink auch basische Bicarbonate, die als natürliche Puffersubstanzen im Körper vorkommen. Die ausgewogene Mineralstoffmischung in BASENTABS pH-balance PASCOE trägt zu einer normalen Muskelfunktion, zur Verringerung von Ermüdung und zum Elektrolytgleichgewicht bei.

Weitere Informationen bei PASCOE Naturmedizin Stefanie Wagner-Suske info@pascoe.de www.pascoe.de

\section{Vitamin D3 Hevert - kleinere Tabletten, neue Rezeptur}

Vitamin D3 Hevert Tabletten (1000 IE Vitamin D) werden seit Anfang Dezember 2015 mit neuer Rezeptur und geändertem Aussehen ausgeliefert. Das Ergebnis der verbesserten Galenik ist eine kleine, schnell auflösende Tablette, wie bisher versehen mit einer Bruchkerbe. Die Tablettengröße konnte deutlich reduziert werden, das Schlucken der Tablette wird erleichtert. Wer die Einnahme in Flüssigkeit bevorzugt, profitiert von dem schnellen Zerfall der Tablette. Durch die regelmäßige Einnahme von Vitamin D3 Hevert wird Mangelerscheinungen und ihren Folgen vorgebeugt. Zusammen mit einer kalziumreichen Ernährung sorgt Vitamin D3 Hevert für ein gesun- des Knochenwachstum und verhindert eine poröse, brüchige Knochenstruktur. Vitamin D3 Hevert ist gut verträglich und auch für Säuglinge und Kleinkinder geeignet. Bereits 1 Tablette täglich kann einem Vitamin-D-Mangel vorbeugen.

Hevert-Arzneimittel GmbH \& Co. KG www.hevert.com

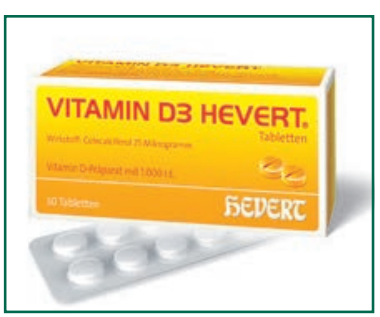

Vitamin D3 Hevert Zusammensetzung: 1 Tablette enthält: Colecalciferol 0,025 mg (entspr. 1000 IE Vitamin D3). Sonstige Bestandteile: Carboxymethylstärke-Natrium (Typ A), DL-alpha-Tocopherol, hochdisperses Siliciumdioxid, hydriertes Sojaöl, Maisstärke, mikrokristalline Cellulose, Natriumstearylfumarat, Gelatine (vom Rind), Sucrose. Anwendungsgebiete: Zur Vorbeugung bei erkennbarem Risiko einer Vitamin D-Mangelerkrankung bei ansonsten Gesunden ohne Resorptionsstörung. Zur unterstützenden Behandlung der Osteoporose. Zur Vorbeugung gegen Rachitis, auch bei Frühgeborenen. Gegenanzeigen: Überempfindlichkeit gegen Colecalciferol oder einen der sonstigen Bestandteile. Hypercalcämie, Hypercalcurie, Pseudohypoparathyreoidismus. Nebenwirkungen: Als Folge der Hypercalcämie bei Überdosierung können akut Herzrhythmusstörungen, Übelkeit, Erbrechen, psychische Symptome, Bewusstseinsstörungen und chronisch vermehrter Harndrang, verstärktes Durstgefühl, Appetitlosigkeit, Gewichtsverlust, Nierensteinbildung, Nierenverkalkung, Verkalkungen in Geweben außerhalb des Knochens auftreten. Zul.-Nr. 6899064.00.00. Packungsgrößen: 50, 100, 200 Tabletten. 